\title{
The evolution and revival structure of angular momentum quantum wave packets (Tutorial)
}

\author{
Marcis Auzinsh \\ Department of Physics, \\ University of Latvia, Rainis blvd. 19, \\ Riga LV-1586, Latvia
}

\begin{abstract}
In this paper a coherent superposition of angular momentum states created by absorption of polarized light by molecules is analyzed. Attention is paid to the time evolution of wave packets representing spatial orientation of internuclear axis of diatomic molecule. Two examples are considered in detail. Molecules absorbing light in a permanent magnetic field experiencing Zeeman effect and molecules absorbing light in a permanent electric field experiencing quadratic Stark effect. In a magnetic field we have a wave packet that evolves in time exactly as classical dipole oscillator in a permanent magnetic field. In the second case we have the wave packet that goes through periodical changes of a shape of the packet and revivals of initial shape. This is a pure quantum behavior. Classical motion of angular momentum in an electric field in case of quadratic Stark effect is known to be aperiodic. Obtained solutions for wave packet evolution are briefly compared with Rydberg state coherent wave packets and harmonic oscillator wave packets.
\end{abstract}

\section{INTRODUCTION}

There are some problems that can be found in every classical mechanics text book. For example, rotation of the planets around the Sun under the action of the gravitation force or the oscillations of the pendulum under the action of the quasielastic force.

In quantum mechanics there are very similar problems of the same importance. The motion of the electron around the nucleus under the action of Coulomb force or the vibration of a diatomic molecule along the line connecting both nuclei. To compare these two sets of problems from which the first belongs to the macroscopic world and the other to the microscopic world one can ask the questions of a type: Is it possible to observe the motion of an electron in a Kepler orbit around the nucleus in the same way as it is possible to observe the motion of the planet around the Sun? That is, is it possible to obtain experimentally a Rutherford atom when electron rotates around the nucleus in a Kepler orbit? Is it possible to observe oscillations of the nuclei in a molecule that are similar to classical oscillations of point particle bound by a quasielastic force? An affirmative answer to these questions, as is well known, is given by the correspondence principle of quantum mechanics [1].

The most common way how we can experimentally examine the objects in the micro world is by their interaction with light. At the same time it is common wisdom, see for example [1], that the methods of ordinary optical spectroscopy generally involve excitation of individual stationary states of atoms and molecules. Such states describe objects that are quantum mechanical by nature. For example, even for arbitrarily large quantum numbers a single stationary state of an electron in a Coulomb field does not come close to describing the motion of a localized particle in a Kepler orbit, just as for any quantum number a stationary wave function of a harmonic oscillator does not describe the harmonic oscillations of a localized particle. In fact, classical motion is never obtained from excitation of single quantum state.

Recently, with the use of ultrashort optical pulses, it has become possible to create coherent superpositions of many quantum states to obtain localized wave packets that are particle-like objects that obey quasiclassical laws, see [2, 3] and references cited therein. Usually electrons moving in orbits with large radiuses and oscilations of molecules are examined in these experiments. The processes with these wave packets usually are very fast - often occurring on a picoor even on a femto-second time scale. These types of processes are most often analyzed to examine correspondence between the classical and quantum description of the objects in the microscopic world.

The interaction of particles with definite angular momentum with electric and magnetic fields provides a second, more accessible, but less exploited, way to examine the correspondence between the quantum and classical nature of the microscopic world. For example, from the view point of classical physics the angular momentum vector of a charged particle spinning in a magnetic field will precess around the field direction with the Larmor frequency [4]

$$
\omega_{L}=\frac{g_{J} \mu_{B} B}{\hbar}
$$

preserving the projection of the angular momentum on the direction of the field. Here $g_{J}$ is a Lande factor, $\mu_{B}$ is the Bohr magneton, and $B$ is the magnetic field strength. To determine the behavior of a particle - atom or molecule - in an external magnetic field in quantum mechanics one must deal with the particle's angular momentum states 
and corresponding wave functions $Y_{J M}(\theta, \varphi)$. To obtain classical-like motion of quantum angular momentum in an external field one must analyze superposition of angular momentum states. For diatomic molecules squared modulus of this superpositional wave packet $\Psi(\theta, \varphi, t)$ will show the probability to find molecular axis with a certain orientation in space. Namely $|\Psi(\theta, \varphi, t)|^{2} \sin \theta d \theta d \varphi$ is a probability to find molecular axis in the direction in space characterized by spherical angles $\theta$ and $\varphi$. For the case of molecules this approach has another advantage. To compare classical and quantum results usually one wants to examine the behavior of the system as the angular momentum become large. According to the correspondence principle these states should behave classically. For the case of rotational states of molecules it is very common and straightforward to create states with large angular momentum quantum numbers, with $J \propto 100$ typical [5]. The situation is different for the case of atomic Rydberg states. It is not at all easy to create in a laboratory states with principal quantum number $n$ close to 100 [2].

Partially the problem to obtain coherent superposition of angular momentum states was solved already many years ago, when quantum beats in an external magnetic field were observed experimentally for the first time by Aleksandrov in Russia [6] and by Dodd with coworkers in United Kingdom [7]. They used light pulse to excite coherently several angular momentum states $|J, M\rangle=Y_{J M}(\theta, \varphi)$ with the same angular momentum quantum number $J$, but different magnetic quantum numbers $M$ simultaneously and coherently. In an external magnetic field these angular momentum states have different energies $E_{M}$ and hence the corresponding wave functions have different phase factors $\exp \left[-i\left(E_{M} / \hbar\right) t\right]$. In an experiment one can observe harmonic time dependencies of polarized fluorescence that corresponds to the beats between these wave functions with different phase factors in the same way as in a signal processing one can observe beets between two or more harmonic signals with slightly different frequencies.

From the practical view point these experiments can be more straightforward than experiments with Rydberg state atoms or instant excitation of oscillations in molecules. An obvious reason for this is that speed of the processes in an external field usually are slower and in any case it can be controlled by controlling external field strength. As a result, for excitation of the state one can use much longer laser pulses and also observation of the state dynamics can be performed by much slower experimental devices [5].

In this paper quantum beat experiments will be analyzed by considering the creation and time evolution of angularly localized wave packets. This unique approach provides an opportunity to examine the correspondence between classical and quantum-mechanical periodic motion as induced by electric and magnetic fields. In our knowledge from these positions these experiments have not been analyzed before.

\section{REVIVAL STRUCTURE OF WAVE PACKETS}

The time-dependant wave functions for angularly localized angular momentum quantum wave packets formed as a coherent superposition of angular momentum eigenstates may be written as

$$
\Psi(\theta, \varphi, t)=\sum_{M} c_{M} Y_{J M}(\theta, \varphi) \exp \left(-i \frac{E_{M}}{\hbar} t\right)
$$

where $Y_{J M}(\theta, \varphi)$ is an ordinary spherical function [8] and the coefficients $c_{M}$ are complex amplitudes.

We would like to examine to what extent and for how long time evolution of this wave packet coincides with the predictions of classical physics. For example, classically, an angular momentum of a rotating rigid charge distribution will precess in the magnetic field with the Larmor frequency $\omega_{L}(1.1)$. If there is a coincidence between the quantum and classical discription, then the position of the "center of gravity" of a spatially localized wave packet (the average value of the particle angular coordinates) must precess in space according to the rules of classical mechanics [4]

$$
\frac{d \mathbf{J}}{d t}=\mu \times \mathbf{B}
$$

Here $\mathbf{J}$ is a classical angular momentum vector, $\mu$ - magnetic dipole moment of particle, $\mathbf{B}$ - strength of the external magnetic field.

Because classical particles have a definite direction of angular momentum, wave packets localized in space usually have $c_{M}$ well centered around some particular mean quantum number $\bar{M}$. For a similar reason wave packets that can be created from stationary atomic Rydberg state wave functions are centered around some definite value $\bar{n}$ of the principle quantum number $n$ of the atomic state. Rydberg wave packets weighted by coefficients possessing Gaussian distribution [9] are particularly well investigated:

$$
\left|c_{n}\right|^{2}=\frac{1}{\sqrt{2 \pi} \sigma} e^{-(n-\bar{n}) / 2 \sigma^{2}} .
$$


Here the parameter $\sigma$ characterizes the width of this distribution. Another particularly well investigated case is the coherent states of a harmonic oscillator that can be obtained from harmonic oscillator wave functions $|v\rangle$ weighted by coefficients in the form 9

$$
c_{v}=e^{-(1 / 2)|\alpha|^{2}} \frac{\alpha^{v}}{\sqrt{v !}}
$$

where $v$ is the vibration quantum number and $\alpha$ is a parameter.

What we are interested in most, when we think about evolution of wave packets is, what are the laws governing the long-term postclassical evolution of wave packets beyond the bounds of the dynamics according to the correspondence principle?

The assumption that the weighting probabilities $\left|c_{M}\right|^{2}$ are strongly centered around a mean value $\bar{M}$ (or $\bar{n}, \bar{v}$ ) means that only those states with energies $E_{M}$ near the value $E_{\bar{M}}$ enter appreciably into the sum of Eq. (2.1). This permits an expansion of energy in a Taylor series in $M$ around the centrally excited value $\bar{M}$

$$
E_{M}=E_{\bar{M}}+E^{\prime} \bar{M}(M-\bar{M})+\frac{1}{2} E^{\prime \prime} \bar{M}(M-\bar{M})^{2}+\frac{1}{6} E^{\prime \prime \prime} \bar{M}(M-\bar{M})^{3}+\ldots,
$$

where each prime on $E \bar{M}$ denotes a derivative at point $M=\bar{M}$.

The derivative terms in Eq. (2.5) define distinct time scales [1] 9 []

$$
T_{c l}=\frac{2 \pi}{\left|E^{\prime} \bar{M}\right|}, \quad t_{\text {rev }}=\frac{2 \pi}{\frac{1}{2}\left|E^{\prime \prime} \bar{M}\right|}, \quad t_{s r}=\frac{2 \pi}{\frac{1}{6}\left|E^{\prime \prime \prime} \bar{M}\right|} .
$$

The first time scale $T_{c l}$ is called the classical period. It is the period after which system returns to it's initial position according to the laws of classical physics. The second time scale $t_{r e v}$ is the revival time. This is a time after which the initial wave function will be partially or completely rebuilt. The third time scale $t_{s r}$ is the superrevival time. It represents the time after which the wave function will be rebuilt in case that it is only partially rebuilt after the revival time. For the most commonly investigated situations of the coherent superposition of Rydberg states and anharmonic oscillators the timescales are ordered so that $T_{c l} \ll t_{r e v} \ll t_{s r}$. But, as we will see, this time ordering changes for the coherent superposition of angular momentum states in an electric field.

One particularly good thing about evolution of angular momentum wave packets is that we can know exactly and to some extent, control by changing excitation geometry and polarization of excitation light, the $c_{M}$ distribution that will occur in a realistic experiment.

As an example let us consider a so called $Q$-type of molecular transition when light excites molecules from the ground state to the excited state and both states have the same value of angular momentum quantum number $J$. Let us further assume that the exciting radiation is linearly polarized with the light vector e lying perpendicularly to an external magnetic field B. The probability of finding molecules in a particular angular momentum state $Y_{J M}(\theta, \varphi)$ can be found by determining the diagonal elements of the density matrix $f_{M M}$ which give the population of angular momentum substates characterized by a magnetic quantum number $M$ (for details see Appendix I and 画, 5])

$$
\begin{aligned}
f_{M M} & =\left|c_{M}\right|^{2}=\sum_{\mu}\left|\left\langle M\left|\mathbf{e}^{*} \mathbf{d}\right| \mu\right\rangle\right|^{2}= \\
& =\frac{1}{2}\left[\left(C_{11 J M-1}^{J M}\right)^{2}+\left(C_{1-1 J M+1}^{J M}\right)^{2}\right]=\frac{1}{2}-\frac{M^{2}}{2 J(J+1)} .
\end{aligned}
$$

Here $\left\langle M\left|\mathbf{e}^{*} \mathbf{d}\right| \mu\right\rangle$ is the optical transition matrix element, $\mathbf{d}$ is the optical transition dipole moment operator, $C_{11 J M-1}^{J M}$ is the Clebsch-Gordan coefficient [8], and $\mu$ is a ground state magnetic quantum number. In this expression ClebschGordan coefficients of a type $C_{11 J M-1}^{J M}$ represent quantum mechanical amplitude to excite an angular momentum state $\left|J^{\prime}, M\right\rangle$ from an initial (usually ground) state $\left|J^{\prime \prime}, \mu=M-1\right\rangle$. In this particular case of a $Q$-type molecular transition the absorption of light does not change the angular momentum of the molecule or atom, so $J^{\prime \prime}=J^{\prime}=J$. As an example for $J=20, f_{M M}=\left|c_{M}\right|^{2}$ as calculated from Eq. (2.7) is given in Figure. 1

Off diagonal elements of the density matrix represent the coherence (phase relations) between different angular momenta substates. Off diagonal matrix elements can be calculated as

$$
f_{M M^{\prime}}=\overline{c_{M} c_{M^{\prime}}^{*}}=\sum_{\mu}\left\langle M\left|\mathbf{e}^{*} \mathbf{d}\right| \mu\right\rangle\left\langle M^{\prime}\left|\mathbf{e}^{*} d\right| \mu\right\rangle^{*}
$$

For $Q$-excitation with a pulsed light polarized along $y$ axis besides the diagonal matrix elements calculated according to the Eq. (2.7) we will have the following non-zero offdiagonal matrix elements [4] 


$$
\begin{aligned}
f_{M+1, M-1} & =f_{M-1, M+1}=\frac{1}{2} C_{J M 1-1}^{J M-1} C_{J M 11}^{J M+1} \\
& =-\frac{\sqrt{\left(J^{2}-M^{2}\right)\left[(J+1)^{2}-M^{2}\right]}}{4 J(J+1)} .
\end{aligned}
$$

From the density matrix elements for angular momentum states, we can easily calculate the squared wave function that represents the probability density

$$
|\Psi(\theta, \varphi, t)|^{2}=\frac{3}{2 J+1} \sum_{M M^{\prime}} f_{M M^{\prime}} Y_{J M} Y_{J M^{\prime}}^{*} \exp \left(-i \omega_{M M^{\prime}} t\right)
$$

where $\omega_{M M^{\prime}}=\left(E_{M}-E_{M^{\prime}}\right) / \hbar$.

\section{ANGULAR MOMENTUM WAVE PACKETS IN A MAGNETIC OR AN ELECTRIC FIELD}

\section{A. Atom or molecule in the external magnetic field}

If an atom or molecule is in an external magnetic field experiencing ordinary Zeeman effect the angular momentum state's $J$ magnetic sublevels $|J, M\rangle$ with different magnetic quantum numbers $M$ will have energies

$$
E_{M}(M)=E^{(0)}+g_{J} \mu_{B} B M / \hbar=E^{(0)}+E_{L} M .
$$

Where $E^{(0)}$ is the energy of a state in the absence of the external field. According to Eq. (2.6) we can expect classical period $T_{c l}$ to be equal to $2 \pi / E_{L}=2 \pi /\left(g_{J} \mu_{B} B / \hbar\right)$. All other periods will be infinite. Thus time evolution of this angular momentum wave packet will be infinitely long rotation around the external magnetic field direction with Larmor angular frequency $\omega_{L}=2 \pi / T_{c l}$. No changes apart from the rotation in space around the magnetic field direction will occur to the wave function. This is what actually has been observed experimentally in the past [10] as a harmonic modulation of an intensity of a polarized fluorescence from an ensemble of atoms or molecules excited by a short laser pulse. This is a well known effect of quantum beats induced by magnetic field.

From the view point of an evolution of a wave packet, this result is similar to the well known behavior for coherent states of a harmonic oscillator. In his pioneering paper Ervin Schrodinger wrote as early as in 1926, that wave packets formed as coherent states of harmonic oscillator will oscillate infinitely long between classical turning points without dispersion, for references see [9]. The main reason for this is that the energies of quantum harmonic oscillator states depend linearly on the vibration quantum number $v$. It means that only the first derivative $E^{\prime} \bar{v}$ in the expansion of type (2.5) will differ from zero. In case of Zeeman effect we observe the same linear energy dependence of the magnetic sublevels of atomic or molecular states on the magnetic quantum number $M$. As a result, according to Eq. (2.6) we have infinitely long classical type motion of the wave packet that represent the precession of angular momentum in a magnetic field. One can easily calculate angular momentum distribution after the pulsed excitation following equations (2.8) - (2.10). This result appears to be independent of the value of angular momentum quantum number $J$. It is

$$
|\Psi(\theta, \varphi, t)|^{2}=\frac{3}{8 \pi}\left[1-\sin ^{2} \theta \sin ^{2}\left(\varphi-\omega_{L} t\right)\right] .
$$

We have donut shape wave function that rotates in space around $z$ axis with Larmor frequency $\omega_{L}$, Eq. (1.1) [5], see Fig. 2. The fact that the result is independent of the angular momentum quantum number $J$ is worth to mention. It is interesting especially because this distribution coincides precisely with the result that would appear if we would consider absorption of electric dipole oscillator in classical physics in the same circumstances. Indeed, if instead of considering artificial wave packets that can only be studied theoretically, we consider wave packets that can be obtained in a realistic experiment it is not uncommon for the quantum and classical results coincide even for small quantum numbers, see [5]. In classical approach this donut shape disrtibution of molecular axis can be understood if one keeps in mind that for $Q$-type molecular transition in a classical approach absorbing dipole is oriented along $\mathbf{J}$, it means perpendicularly to the intermolecular axis of a rotationg molecule [5]. 


\section{B. Molecule in an external electric field}

The evolution of a molecular wave packet in an external electric field is quite distinct from the case of a magnetic field. For both cases we have the same amplitudes $c_{M}$ of partial components of wave function in Eq. (2.1) and the same density matrix. But in case of an electric field we will have a different magnetic sublevel energy $E_{M}$ dependence on the magnetic quantum number $M$. Let us consider molecule in a state experiencing a quadratic Stark shift. This is a type of Stark effect most commonly observed with atoms and molecules. In this case we will have an energy dependence on the magnetic quantum number of the form

$$
\begin{aligned}
E_{M}(M) & =E^{(0)}+\frac{d^{2} \mathcal{E}^{2}}{h B}\left[\frac{J(J+1)-3 M^{2}}{2 J(J+1)(2 J-1)(2 J+3)}\right] \\
& =E^{(0)}+E_{\text {Stark }}^{(1)}(\mathcal{E})+E_{\text {Stark }}^{(2)}(\mathcal{E}) M^{2}
\end{aligned}
$$

We know that in case of the Stark effect, the classical motion of angular momentum in an external electric field is aperiodic [4,11. This is exactly what we can see from Eq. (3.3). The first derivative of $E_{\bar{M}}^{\prime}=0$ is zero and that means that $T_{c l}$ is infinite. At the same time the second derivative

$$
E_{\bar{M}}^{\prime \prime}=-\left[\frac{3 d^{2} \mathcal{E}^{2}}{h B J(J+1)(2 J-1)(2 J+3)}\right]
$$

differs from zero and one can expect $t_{r e v}$ be different from zero or infinity. This is exactly what was predicted for Stark quantum beets [12]. Figure 3 depicts one period of evolution of the wave function for state $J=1$ excited by linearly polarized light with e vector lying in $z y$ plane and forming angle $\pi / 4$ with the direction of an external electric field $\mathcal{E}$. The $R$-type $(J=0 \longrightarrow J=1)$ optical transition is assumed. The analytical expression describing probability density on Figure 3 is

$$
|\Psi(\theta, \varphi, t)|^{2}=\frac{3}{8 \pi}\left\{1-\sin ^{2} \theta \cos ^{2} \varphi+\sin 2 \theta \sin 2 \varphi \cos \left[\left(2 \pi / t_{\text {rev }}\right) t\right]\right\} .
$$

It can be calculated using formulae (2.8), 2.10) and Appendix.

The interesting feature of Stark effect is that revival time

$$
t_{r e v}=\frac{4 \pi h B J(J+1)(2 J-1)(2 J+3)}{3 d^{2} \mathcal{E}^{2}}
$$

approaches infinity when angular momentum approaches infinity, it means a particle with very large angular momentum starts to behave truly classically. This quantum mechanical revival is not only interesting as a peculiar behavior of wave function, but as well can be used to orient molecules in beams effectively 13. Probably other applications of periodic behavior of atomic and molecular wave function in electric field can be foreseen.

\section{SUMMARY}

This paper points attention and illustrates two examples of angular momentum coherent wave packets that are of grate interest due to their peculiar properties. They are less noticed than they deserve. These wave packets describe precession of internuclear axes of diatomic molecules in an external field.

The first example was the angular momentum coherent superposition state created by the absorption of polarized light by a molecule in an external magnetic field. There are many examples of such wave packets created in experiments for states of diatomic molecules [10]. But never has this situation of a coherent superposition of angular momentum eigenstates been analyzed with the same machinery used to analyze the coherent superposition of Rydbaerg states or coherent states of harmonic oscillator. In experiments with molecules absorbing light in permanent external fields very often states with large rotational angular momentum quantum numbers of $J \propto 100$ were involved. This allows us to compare these states with behavior of a spinning particle in an external field.

It is known that classical angular momentum in an external magnetic field will precess around the magnetic field direction with Larmor frequency $\omega_{L}$.

Quantum wave packet in a magnetic field will experience the same motion. Period of rotation of wave packet will coincide perfectly with period of precession of classical angular momentum. Wave packet will last for ever (actually as long as the excited state of molecule will live). It will not undergo any disintegration. 
There is known only one other example when the wave packet evolves in time without dispersion. It is a coherent state of harmonic oscillator [9]. The reason for this type of motion in both cases is the same. All coherently excited wave functions in these examples represent states that are equally separated in energy scale, i.e. the systems have energy levels with equally separated steps.

Another example considered here was angular momentum states in an external electric field causing a quadratic Stark effect. In this case an ensemble of angular momentum will evolve aperiodically in classical physics 4 . In quantum physics we will have periodical motion during which the wave function will periodically disintegrate and than, after a definite period, will go through a revival.

This is quite unique dynamics. It is more usual for systems to have a period of classical motion $T_{c l}$ that is substantially shorter than revival time $t_{r e v}$, as is true for the Rydberg states or anharmonic oscillator states. It means that during one revival period this type quantum system will undergo many classical periods. In the example of the quadratic Stark effect in an external electric field, we have exactly the opposite extreme. The system has no classical period at all. From the view point of classical physics the system is aperiodic. But quantum evolution of the wave function still have a well defined period which becomes longer and longer, when angular moment of system increases and system approaches classical limit.

\section{APPENDIX I. CALCULATION OF DENSITY MATRIX ELEMENTS.}

Calculation of density matrix elements entering Eqs. 2.7) and (2.8)

$$
f_{M M^{\prime}}=\sum_{\mu}\left\langle M\left|\mathbf{e}^{*} \mathbf{d}\right| \mu\right\rangle\left\langle M^{\prime}\left|\mathbf{e}^{*} d\right| \mu\right\rangle^{*}
$$

mainly consist in calculation of quantum mechanical matrix elements of a type

$$
\left\langle M\left|\mathbf{e}^{*} \mathbf{d}\right| \mu\right\rangle .
$$

Let us now have a look in more detail at how these matrix elements can be calculated. The first step is to calculate the Hermitian product [14] $\left(\mathbf{e}^{*} \cdot \mathbf{d}\right)$ of light polarization vector $\mathbf{e}$ and optical transition dipole moment $\mathbf{d}$. Meaning of this product is very close to that of a scalar or dot product of two ordinary vectors. Only in this case we are dealing with complex vectors and to find the projection of one vector onto another or - which is the same - to find "how much of one vector is contained into another" we must calculate a Hermitian product.

We are using here complex vectors, because it is a simple way to describe rotations in quantum mechanics as well as in classical physics. Let us see how these complex vectors and their components in a cyclic system of coordinates [5.8 can be used to describe, for example, the polarization of light. An arbitrary light polarization vector (unit vector $\mathbf{e}$ in the direction of the electric field vector of the light wave) in cyclic coordinates can be written as

$$
\begin{aligned}
e^{+1} & =-\frac{1}{\sqrt{2}}\left(e_{x}-i e_{y}\right), \\
e^{0} & =e_{z}, \\
e^{-1} & =\frac{1}{\sqrt{2}}\left(e_{x}+i e_{y}\right) .
\end{aligned}
$$

If we multiply now these vector components by a phase factor exp $(-i \Omega t)$ that represents the oscillations of electric field in light wave, we can easily see, that for

$$
\begin{aligned}
e^{+1} \exp (-i \Omega t) & =-1 / \sqrt{2}\left[e_{x} \exp (-i \Omega t)-i e_{y} \exp (-i \Omega t)\right] \\
& =-1 / \sqrt{2}\left\{e_{x} \exp (-i \Omega t)+e_{y} \exp [-i(\Omega t+\pi / 2)]\right\}
\end{aligned}
$$

oscillations along $x$ axis are quarter period ahead of those along $y$ axis and this means that this component of the e vector represents lefthanded circular polarized in $x y$ plane light beam that propagates in the positive direction of $z$ axis. It means that a light wave in which electric field vector rotates counterclockwise is viewed in such way that the radiation approaches the observer in the positive direction of the $z$ axis.

In a similar way one can see that the $e^{-1}$ component represents righthanded circular polarized light that propagates along $z$ axis with the e vector rotating in a clockwise direction in the $x y$ plane.

And finally, the $e^{0}$ component represents linearly along $z$ axis polarized light for which e oscillates along $z$ axis. Just as light e vector can be represent by its three Cartesian components, every vector can be represented by its three 
cyclic components. In case of light polarization vector (and many other vectors in quantum mechanics) these cyclic components are more practical for calculation than the Cartesian representation.

Now let us turn back to the matrix elements of a type (5.2). According to vector algebra for cyclic vectors, the Hermitian product can be written as [5,8]

$$
\left\langle M\left|\mathbf{e}^{*} \mathbf{d}\right| \mu\right\rangle=\sum_{q}\left(e^{q}\right)^{*}\left\langle M\left|d^{q}\right| \mu\right\rangle .
$$

Further for a matrix element $\left\langle M\left|d^{q}\right| \mu\right\rangle$ we can apply Wigner-Eckart theorem [5, ק]. It allows to separate angular and dynamical part of this matrix element. What does this mean? For example, in classical physics if one wants to know how effectively an oscillating electric field e can excite linear dipole oscillator $\mathbf{d}$ one must calculate scalar product $(\mathbf{e} \cdot \mathbf{d})=e d \cos (\widehat{e d})$. It means that we can separate dynamic part $e d$ that describes the vector magnitude, and the angular part cos $(\widehat{e d})$ that represent their mutual orientation. In the same way in quantum physics Wigner-Eckart theorem allows us to achieve the same separation for optical transition from initial state $\left|J^{\prime \prime} \mu\right\rangle$ to excited state $\left|J^{\prime} M\right\rangle$. Namely

$$
\left\langle M\left|d^{q}\right| \mu\right\rangle=\frac{1}{\sqrt{2 J^{\prime}+1}} C_{J^{\prime \prime} \mu 1 q}^{J^{\prime} M}\left\langle J^{\prime}\|d\| J^{\prime \prime}\right\rangle,
$$

where $C_{J^{\prime \prime} \mu 1 q}^{J^{\prime} M}$ is a Clebsch-Gordan coefficient and $\left\langle J^{\prime}\|d\| J^{\prime \prime}\right\rangle$ is a reduced matrix element, that represents the dynamical part of optical transition probability. It is obvious that the Clebsch-Gordan coefficient has a numerical value that depends on the values of angular momentum projections $M$ and $\mu$ on the quantization axis $z$, and therefore it describes the angular momentum orientation in space. This is the angular part of the transition probability.

If we now collect all these formulae together we can obtain the final expression that allows us to calculate easily matrix elements (5.1) for arbitrary light polarization and optical transition between arbitrary states

$$
f_{M M^{\prime}}=N \sum_{\mu q_{1} q_{2}}\left(e^{q_{1}}\right)^{*}\left(e^{q_{2}}\right) C_{J^{\prime \prime} \mu 1 q_{1}}^{J^{\prime} M} C_{J^{\prime \prime} \mu 1 q_{2}}^{J^{\prime} M^{\prime}} .
$$

In this last expression a proportionality coefficient $N$ that is insignificant for the purpose of this paper, is not determined.

\section{ACKNOWLEDGMENTS}

The support from National Research Council Twinning Program, Grant No. NP-NRC-6224 is greatly acknowledged. I am very grateful to Prof. Neil Shafer-Ray for a fruitful discussions and University of Oklahoma for the hospitality.

[1] Averbukh I. Sh., Perel'man N.F., "The dynamics of wave packets of highly-excited states of atoms and molecules", Sov. Phys. Usp. 34 (7), 572-591 (1991)

[2] Alberg G. and Zoller P., "Laser Excitation of Electronic wave packets in Rydberg atoms", Phys. Rep. 199, 231-280 (1991)

[3] Garraway B.M. and Suominen K-A, "Wave-packet dynamics: new physics and chemistry in femto-time", Rep. Prog. Phys. 58, 365-419 (1995)

[4] Auzinsh Marcis, "Angular momenta dynamics in magnetic and electric field: Classical and quantum approach", Can. J. Phys. 75, 853-872 (1997)

[5] Auzinsh M. and Ferber R., Optical polarization of Molecules (Cambridge University Press, Cambridge, U.K. 1995), p. 306

[6] Aleksandrov E.B., "Quantum beats of luminescence under modulated light excitation", Opt. Spectrosc. (USSR), 14, 233$234(1963)$

[7] Dodd J.N., Kaul R.D. and Warington D.M., "The modulation of resonance fluorescence excited by pulsed light", Proc. Phys. Soc. London, Sect. A, 84, 176-178 (1964) 
[8] Varshalovich D.A., Moskalev A.N., Khersonskii V.K., Quantum Theory of Angular Momentum (World Scientific, Singapore, 1988), p. 514.

[9] Bluhm Robert, Kostelecky V.Alan, Porter James A, "The evolution and revival structure of localized quantum wave packets", Am. J. Phys. 64 (7), 944-953 (1996)

[10] Hack E. and Huber J.R., "Quantum beat specrtoscopy of molecules", International Reviews in Physical Chemistry, 10, 287-317 (1991)

[11] Hilborn R.C., "Atoms in orthogonal electric and magnetic fields: A cpmparison of quantum and classical models", Am. J. Phys. 63, 330-338 (1995)

[12] Auzinsh M., Ferber R. and Stolyarov A., "Separation of quadratic and linear external field effects in high $J$ quantum beats", J. Chem. Phys. 101 (7), 5559-5565 (1994)

[13] Auzinsh M.P., Ferber R.S., "J-selective Stark orientation of molecular rotation in a beam", Phys. Rev. Lett., 69, 3463-3466 (1992)

[14] Fano U. and Racah G., Irreducible tensorial sets (Academic Press, New York, 1959), p. 169. 


\section{FIGURE CAPTIONS}

\section{Figure 1}

Relative population distribution among magnetic substates $|J, M\rangle$ for angular momentum state with $J=20$.

\section{Figure 2}

Wave function evolution in an external magnetic field. $1-\omega_{L} t=0,2-\omega_{L} t=\pi / 4,3-\omega_{L} t=\pi / 2,4-\omega_{L} t=3 / 4 \pi$, $5-\omega_{L} t=\pi, 6-\omega_{L} t=5 \pi / 4$

Figure 3

Stark quantum beats. $1-t=0,2-t=\frac{1}{5} t_{r e}, 3-t=\frac{1}{4} t_{r e}, 4-t=\frac{1}{2} t_{r e}, 5-t=\frac{3}{4} t_{r e}, 6-t=t_{r e}$ 


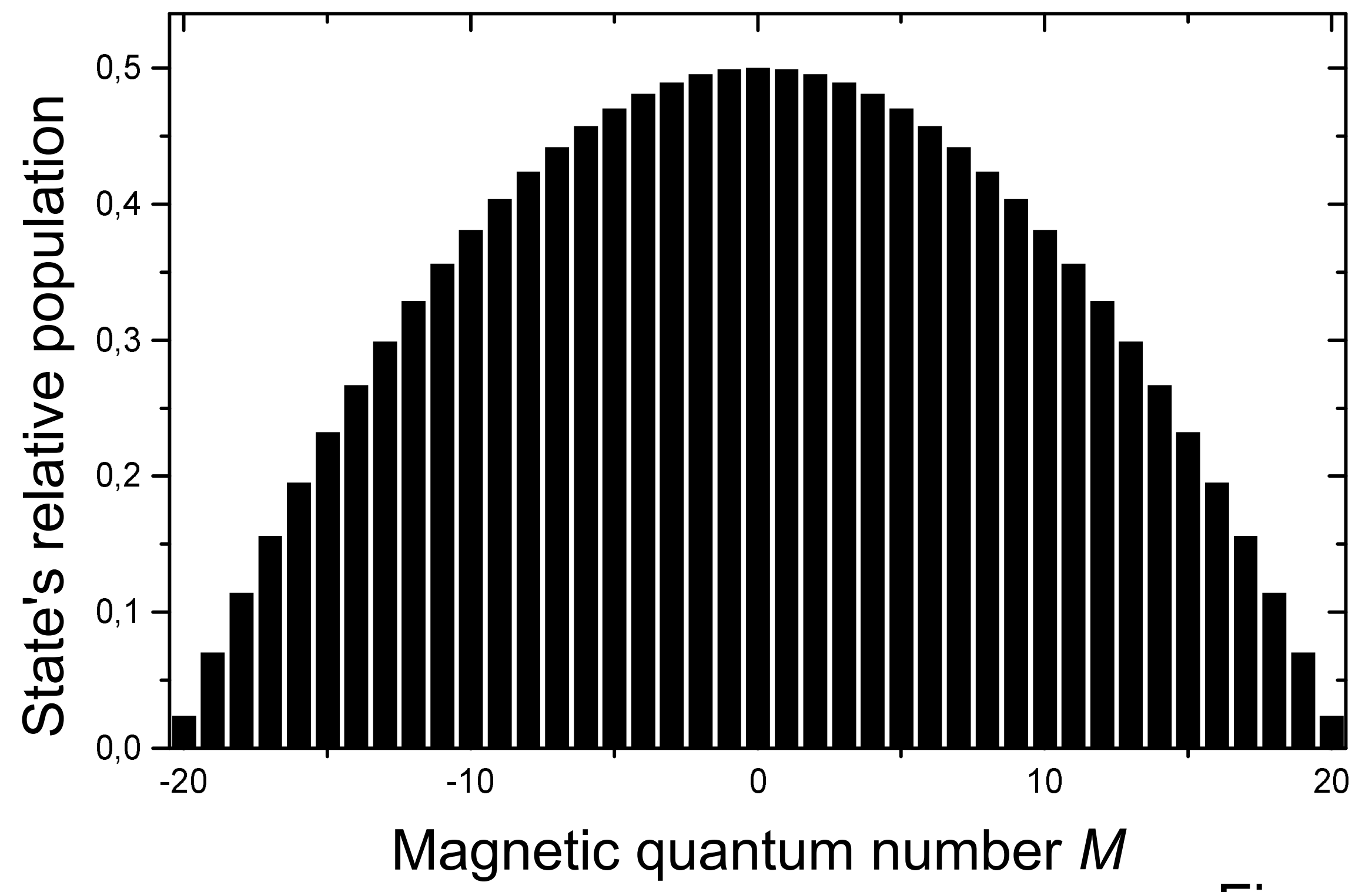

Figure 1 
Figure 2, 1

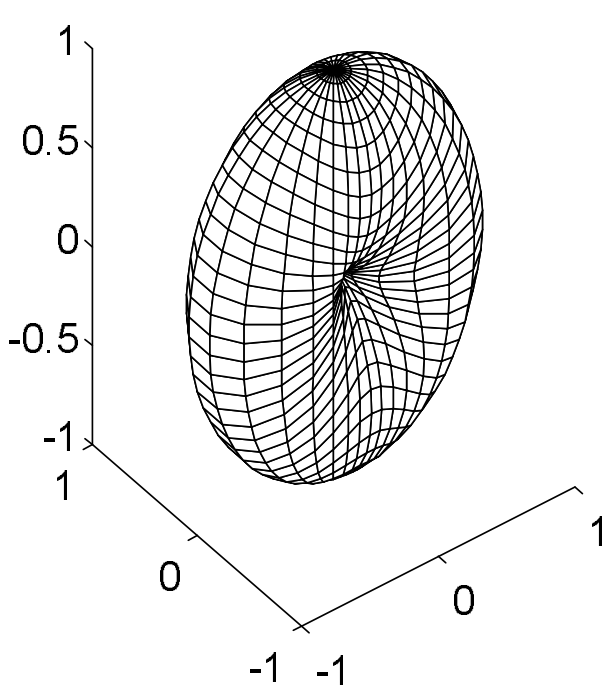

4

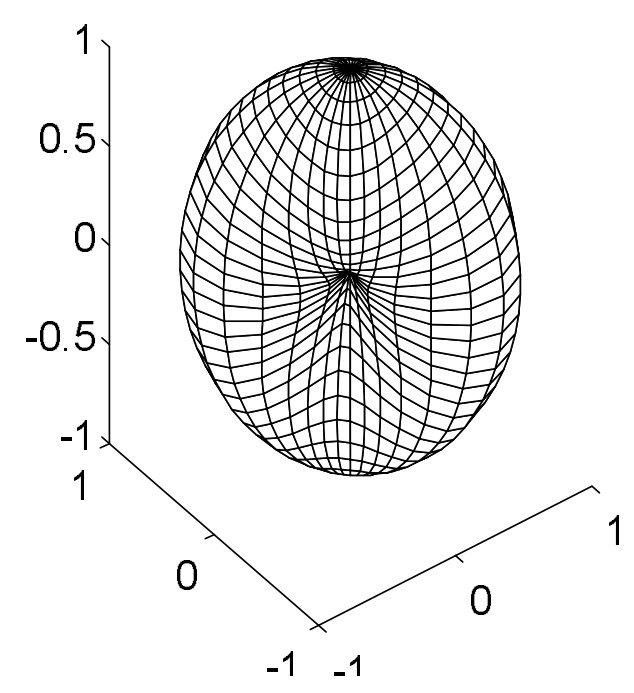

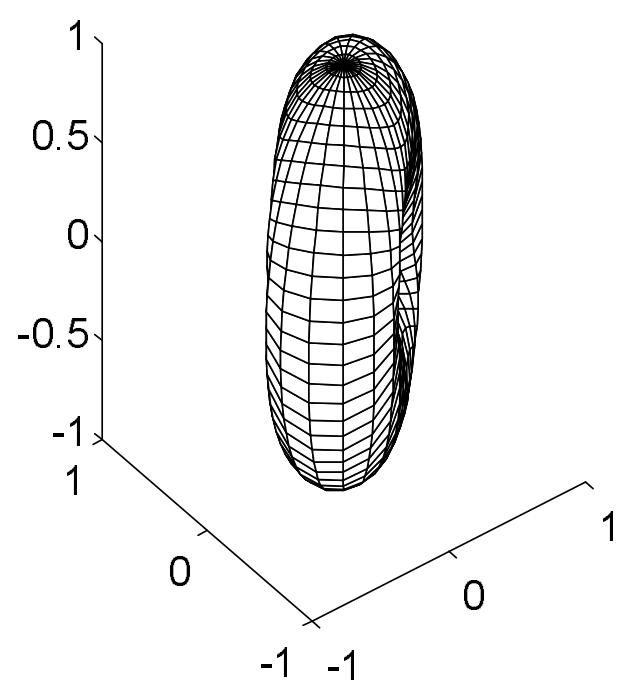

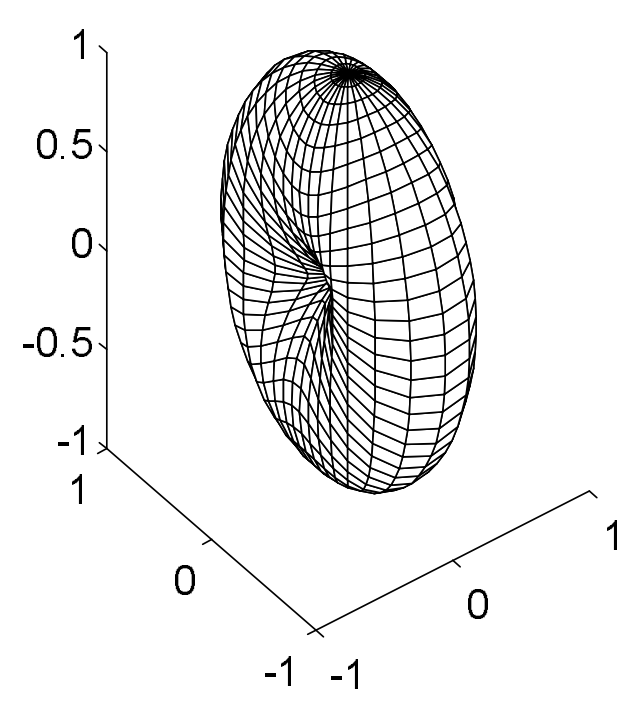

5

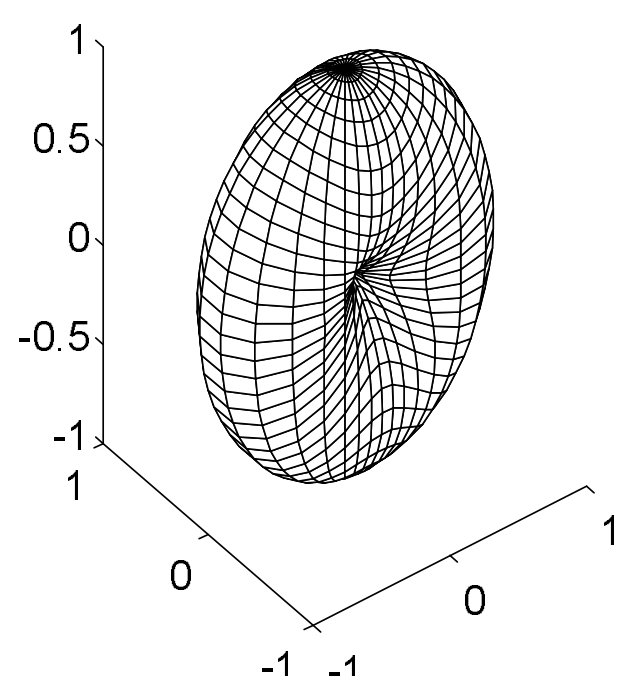

6

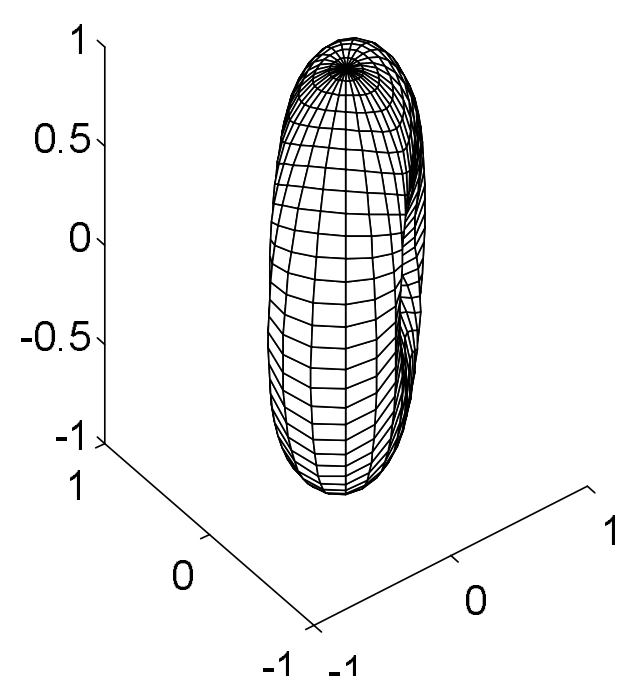


Figure 3, 1

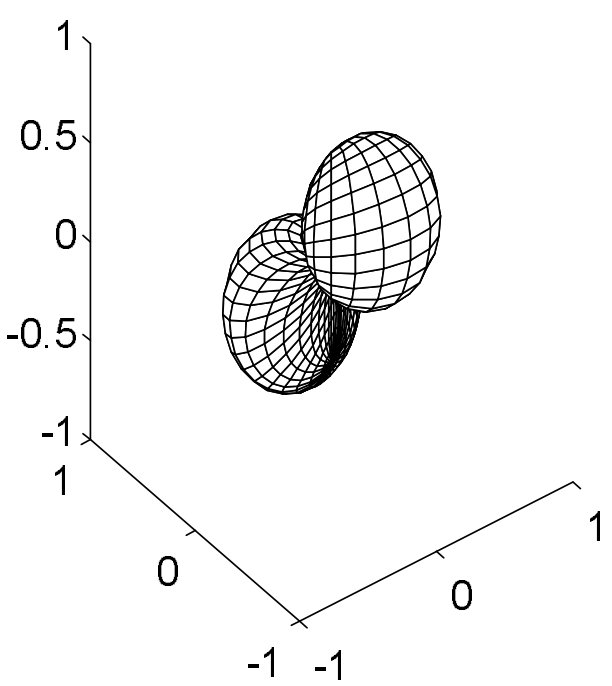

4

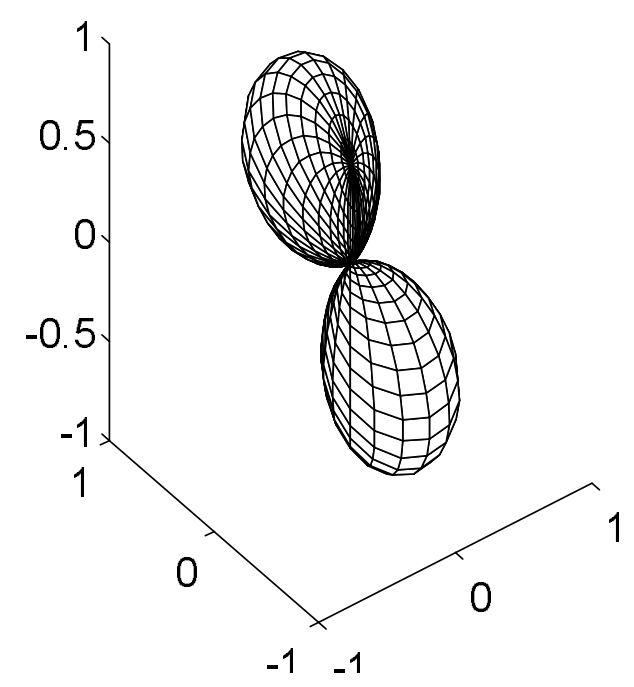

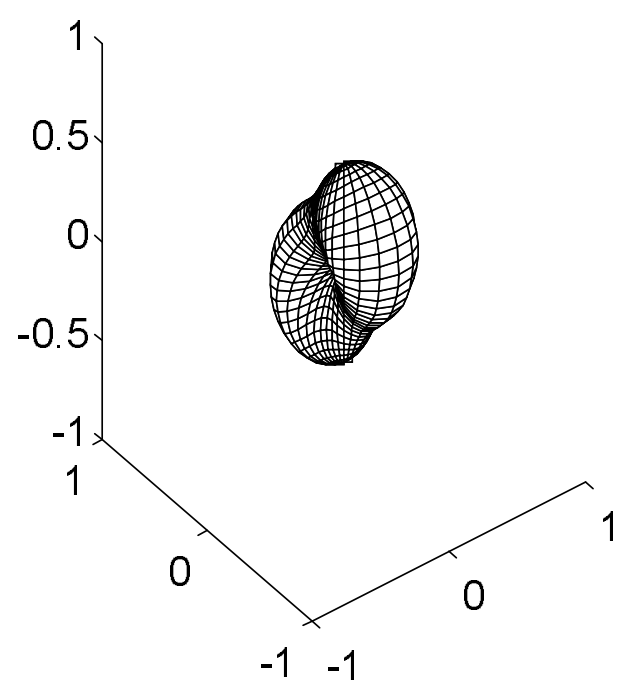

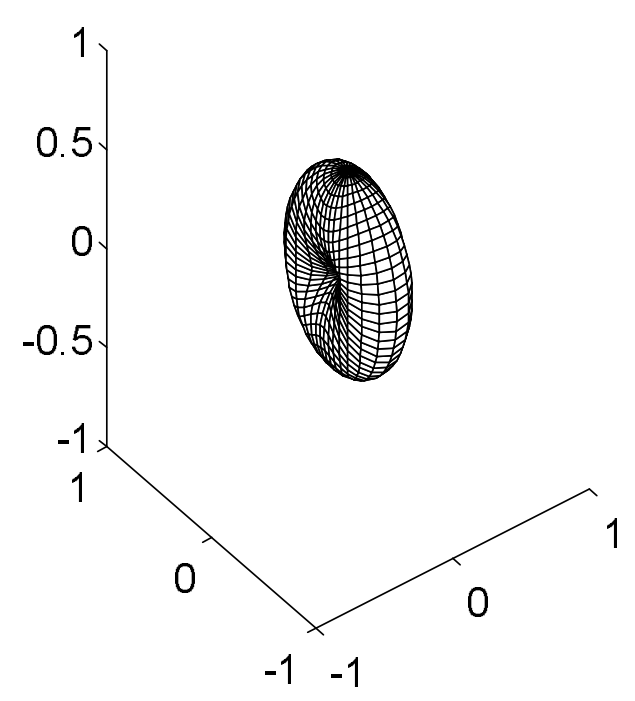

5

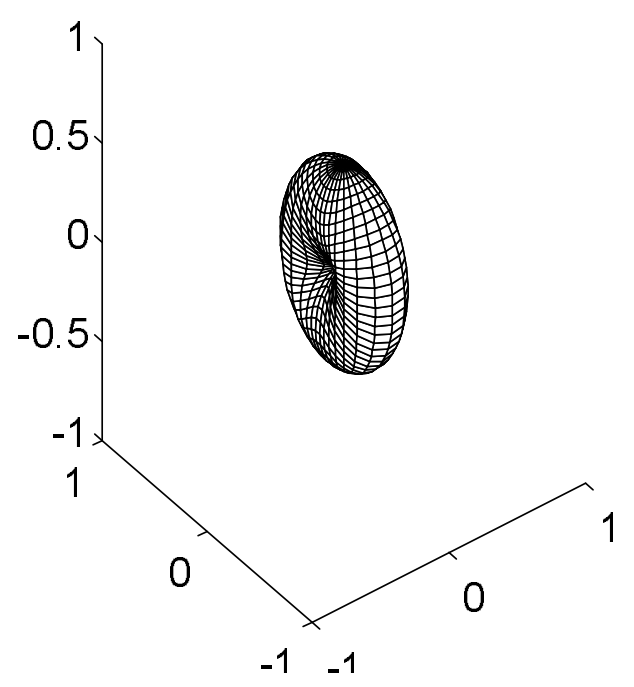

6

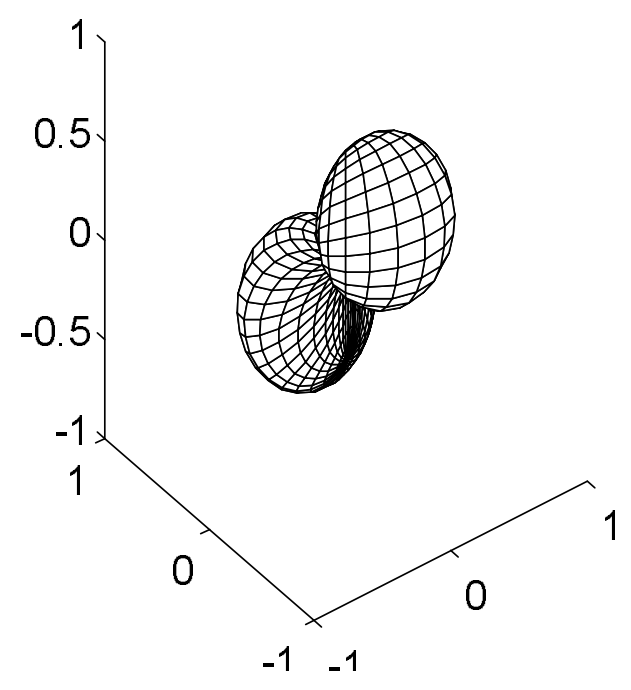

